\title{
Anisotropic electrical conductivity of magnetized hot quark matter
}

\author{
Aritra Bandyopadhyay $\odot,{ }^{1,2}$ Sabyasachi Ghosh $\odot,{ }^{3}$ Ricardo L. S. Farias $\odot,{ }^{1}$ Jayanta Dey $\odot,{ }^{3}$ and Gastão Krein ${ }^{4}$ \\ ${ }^{1}$ Departamento de Física, Universidade Federal de Santa Maria, Santa Maria, RS 97105-900, Brazil \\ ${ }^{2}$ Guangdong Provincial Key Laboratory of Nuclear Science, Institute of Quantum Matter, \\ South China Normal University, Guangzhou 510006, China \\ ${ }^{3}$ Indian Institute of Technology Bhilai, GEC Campus, Sejbahar, Raipur 492015, Chhattisgarh, India \\ ${ }^{4}$ Instituto de Física Teórica, Universidade Estadual Paulista, Rua Dr. Bento Teobaldo Ferraz, \\ 271 - Bloco II, 01140-070 São Paulo, SP, Brazil
}

(Received 27 November 2019; revised 21 September 2020; accepted 17 November 2020; published 8 December 2020)

\begin{abstract}
We studied the effect of a strong magnetic field $(B)$ on the electrical conductivity of hot quark matter. The electrical conductivity is a key transport coefficient determining the time dependence and strength of magnetic fields generated in a relativistic heavy-ion collision. A magnetic field induces Hall anisotropic conduction, phase-space Landau-level quantization and, if sufficiently strong, interferes with prominent QCD phenomena such as dynamical quark mass generation, likely affecting the quark matter electrical conductivity, which depends strongly on the quark masses. To address these issues, we used a quasiparticle description of quark matter in which the electric charge carriers are constituent quarks with temperatureand magnetic-field-dependent masses predicted by a Nambu-Jona-Lasinio model. The model accurately describes recent lattice QCD results showing magnetic catalysis at low temperatures and inverse magnetic catalysis at temperatures close to the pseudocritical temperature $\left(T_{\mathrm{pc}}\right)$ of the QCD phase transition. We found that the magnetic field increases the conductivity component parallel to it and decreases the transverse component, in qualitative agreement with recent lattice QCD results. In addition, we found that (1) the space anisotropy of the conductivity increases with $B$, (2) the longitudinal conductivity increases due to phase-space Landau-level quantization, (3) a lowest Landau level approximation behaves poorly for temperatures close to $T_{\mathrm{pc}}$, and (5) inverse magnetic catalysis leaves a distinctive signal in all components of the conductivity, a prominent peak at $T_{\mathrm{pc}}$. Our study adds to the existing body of work on the hot quark matter electrical conductivity by incorporating nontrivial temperature and magnetic field effects on dynamical mass generation. Our results are useful both for studies employing magnetohydrodynamics simulations of heavy-ion collisions and for getting insight on lattice QCD results.
\end{abstract}

DOI: 10.1103/PhysRevD.102.114015

\section{INTRODUCTION}

Relativistic heavy-ion collisions can produce strong magnetic fields $[1,2]$. Field strengths comparable to or even larger than the strong-interaction scale $\Lambda_{\mathrm{QCD}} \simeq$ $0.25 \mathrm{GeV}$ can be produced in the collisions. Indeed, field strengths as large as $e B \sim 15 m_{\pi}^{2} \gg \Lambda_{\mathrm{QCD}}^{2}$ have been estimated [3] for $\mathrm{Pb}-\mathrm{Pb}$ collisions at the Large Hadron Collider. Such strong fields interfere with prominent stronginteraction phenomena such as dynamical mass generation and the chiral anomaly taking place in the hot quark matter-or quark-gluon plasma. Such interferences are predicted to lead to interesting effects, some of which

Published by the American Physical Society under the terms of the Creative Commons Attribution 4.0 International license. Further distribution of this work must maintain attribution to the author(s) and the published article's title, journal citation, and DOI. Funded by SCOAP ${ }^{3}$. were already observed but the interpretation of the observations is ambiguous because alternative explanations are possible-Refs. [4-7] are recent reviews on this. An adversity here is that the fields are created early in the collision and weaken fast as the system expands $[3,8]$. On the other hand, the early produced fields can induce electric currents in the expanding matter which in turn produce magnetic fields that can last while the system exists [9-12]. A key physical property in the electric-current induction is the electrical conductivity, a quantity poorly constrained by the fundamental theory, quantum chromodynamics (QCD).

The quark matter electrical conductivity is poorly constrained by QCD due to two main reasons: the matter produced in a heavy-ion collision is a strongly interacting many-body system, and a strong magnetic field modifies intrinsic properties of the electric charge carriers in the system. A proper treatment of both features requires nonperturbative methods. Lattice QCD, a nonperturbative first-principles numerical method formulated in Euclidean 
space, can compute the electrical conductivity-and other transport properties such as shear and bulk viscosities-but the computation requires reconstructing spectral functions from Euclidean correlation functions using ill-posed inversion methods from imaginary time to frequency space. For $B=0$, the first lattices results for the conductivity in full QCD appeared only recently [13,14], although results from quenched simulations are available for some time [15-17]. For $B \neq 0$, there are results from quenched simulations of an SU(2) gauge theory [18] and from simulations in full QCD with $N_{f}=2+1$ flavors reported in a very recent preprint [19]. Recent analytical nonperturbative calculations of the conductivity, taking into account effects of the magnetic field, have been performed using different phenomenological approaches [20-29].

Prominent magnetic field effects, however, are neglected in previous studies, namely those that affect intrinsic properties of the electric charge carriers in the medium, which are predominantly the light $u$ and $d$ quarks. This is an important omission: recent lattice QCD results [30-33] have shown that strong magnetic fields have dramatic effects on the QCD phase diagram, notably in the region close to the pseudocritical temperature $T_{\mathrm{pc}} \simeq 0.170 \mathrm{GeV}$, the region associated with the hadron-to-quark transition. The effects are most striking on the $u$ and $d$ quark condensates, namely the condensates increase with the magnetic field for low temperatures and decrease for temperatures close to $T_{\mathrm{pc}}$; in the first case one refers to magnetic catalysis (MC) and in the latter to inverse magnetic catalysis (IMC)—Refs. [34-36] are recent reviews which contain extensive lists of references on this subject. The quark condensates have a direct impact on the effective masses of the $u$ and $d$ quarks, which in turn play an important role in the conductivity.

In the present work we fill this gap in the study of the electrical conductivity of magnetized quark matter by using a quasiparticle model. Much of our understanding of the low-energy regime of QCD, and of the QCD phase diagram in particular, is built on insights gained with quasiparticle models. Among the several existing quasiparticle models, those based on the Nambu-Jona-Lasinio (NJL) model [37,38] have been valuable in this respect-Refs. [3942] are extensive reviews on the model in different QCD applications. In these models, the primary electric charge carriers are the $u$ and $d$ quarks and their response to electromagnetic forces are strongly dependent on their effective in-medium masses. The effective quark masses are determined by the in-medium quark condensate. As mentioned, strong magnetic fields change the condensate and in the present paper take this effect into account in the computation of the electrical conductivity. We use the NJL model of Refs. [43,44], a model that reproduces the lattice QCD data for the quark condensates, showing $\mathrm{MC}$ at low temperatures and IMC at temperatures close to $T_{\mathrm{pc}}$. We derive the expressions of the components of the conductivity by solving the Boltzmann equation in the relaxation-time approximation.

For a realistic quantification of magnetic fields effects in a heavy-ion collision, it is imperative to take into account the temperature and magnetic field dependence of the electrical conductivity. Simulations of the field dynamics invariably involve solving relativistic magnetohydrodynamics equations. These equations also require other transport coefficients, as shear and bulk viscosities. The magnetic field dependence of the shear viscosity was computed recently in Refs. [45-54] and of the bulk viscosity in Refs. [55-59]. Our study of the electrical conductivity adds to this body of work by incorporating important effects neglected in previous studies. Our results should be useful for studies employing magnetohydrodynamics simulations of heavy-ion collisions and for getting insight on lattice QCD results.

The paper is organized as follows. In Sec. II we start reviewing the NJL model quasiparticle description of magnetized quark matter, with particular emphasis on the magnetic catalysis and inverse magnetic catalysis of the chiral condensate. Then we present the derivation of the electrical conductivity by solving the Boltzmann equation in the relaxation time approximation. In Sec. III we present numerical results for the conductivity and discuss their meaning. In particular, we discuss the implications of the changes in the quark masses on two well-known magnetic field effects on the conductivity: breaking of the space isotropy of the conductivity, and dimensional reduction of the dynamics along with quantization of phase space (Landau levels). The first happens when mutually perpendicular electric and magnetic fields exist in the conducting medium, field configurations that actually can be generated in a heavy-ion collision $[4,9]$. The second is a quantum mechanical effect that is particularly important for strong fields. Section IV presents a summary and the perspectives of our study.

\section{FORMALISM}

We start with a brief review of the NJL model quasiparticle description of quark matter at finite temperature $T$ and in the presence of a magnetic field with strength $B$. We focus on the implications of the inverse magnetic catalysis (IMC) phenomenon on the quasiparticle effective mass $M$, the constituent quark mass. Next, we present the derivation of the electrical conductivity within the relaxation time approximation. The main input from the NJL model in these derivations is the $T-$ and $B$-dependent constituent quark mass $M$.

\section{A. Quark matter in presence of a magnetic field}

The Lagrangian density for the isospin-symmetric twoflavor version of NJL model in presence of an electromagnetic field $\left(A^{\mu}\right)$ is given by 


$$
\mathcal{L}_{\mathrm{NJL}}=-\frac{1}{4} F^{\mu \nu} F_{\mu \nu}+\bar{\psi}(\not D-m) \psi+G\left[(\bar{\psi} \psi)^{2}+\left(\bar{\psi} i \gamma_{5} \tau \psi\right)^{2}\right],
$$

where $\psi$ is a flavor doublet of $u$ and $d$ quark fields, each being an $N_{c}$-plet, where $N_{c}=3$ is the number of colors, $m=\operatorname{diag}(u, d)$ the quark-mass matrix, $D_{\mu}=i \partial_{\mu}-Q A_{\mu}$ the covariant derivative, $Q=\operatorname{diag}\left(q_{u}=2 e / 3, q_{d}=-e / 3\right)$ the charge matrix, $A_{\mu}$ the electromagnetic gauge field, $F_{\mu \nu}=\partial_{\mu} A_{\nu}-\partial_{\nu} A_{\mu}$, and $\tau=\left(\tau^{1}, \tau^{2}, \tau^{3}\right)$ are the isospin Pauli matrices. We work in the approximation of exact isospin symmetry, i.e., $m_{u}=m_{d}$. The model is solved in the quasiparticle approximation or, equivalently, in the mean-field approximation, which corresponds to the leading-order approximation in the $1 / N_{c}$ expansion. Since the model is unrenormalizable due to the quadratic fermionic interaction, a regularization procedure must be employed. In the present paper we employ a sharp cutoff $\Lambda$ to regularize ultraviolet divergences.

In the quasiparticle approximation, the gap equation for the constituent quark mass $M$ at finite temperature $T$ and in the presence of a magnetic field $B$ is given by

$$
M=m-2 G \sum_{f=u, d}\left\langle\bar{\psi}_{f} \psi_{f}\right\rangle,
$$

where $\left\langle\bar{\psi}_{f} \psi_{f}\right\rangle$ represents the quark condensate of flavor $f$, which can be written as a sum of three contributions $[43,60,61]$,

$$
\left\langle\bar{\psi}_{f} \psi_{f}\right\rangle=\left\langle\bar{\psi}_{f} \psi_{f}\right\rangle^{\mathrm{vac}}+\left\langle\bar{\psi}_{f} \psi_{f}\right\rangle^{B}+\left\langle\bar{\psi}_{f} \psi_{f}\right\rangle^{T, B},
$$

with

$$
\begin{aligned}
&\left\langle\bar{\psi}_{f} \psi_{f}\right\rangle^{\mathrm{vac}} \\
&=-\frac{M N_{c}}{2 \pi^{2}}\left[\Lambda \sqrt{\Lambda^{2}+M^{2}}-M^{2} \ln \left(\frac{\Lambda+\sqrt{\Lambda^{2}+M^{2}}}{M}\right)\right], \\
&\left\langle\bar{\psi}_{f} \psi_{f}\right\rangle^{B}=-\frac{M\left|q_{f}\right| B N_{c}}{2 \pi^{2}}\left[\ln \Gamma\left(x_{f}\right)-\frac{1}{2} \ln (2 \pi)\right. \\
&\left.+x_{f}-\frac{1}{2}\left(2 x_{f}-1\right) \ln \left(x_{f}\right)\right], \\
&\left\langle\bar{\psi}_{f} \psi_{f}\right\rangle^{T, B}= \sum_{k=0}^{\infty} \alpha_{k} \frac{M\left|q_{f}\right| B N_{c}}{2 \pi^{2}} \int_{-\infty}^{\infty} d p_{z} \frac{n_{F}\left(\omega_{f}\right)}{\omega_{f}},
\end{aligned}
$$

where $q_{f}$ is the electric charge of the quark with flavor $f=(u, d), \Gamma\left(x_{f}\right)$ is the Euler gamma function, $x_{f}=$ $M^{2} / 2\left|q_{f}\right| B$. In addition, in Eq. (6) $k$ indexes Landau levels, with $\alpha_{k}=2-\delta_{k, 0}$ being spin degeneracy, and $n_{F}\left(\omega_{f}\right)$ is the Fermi-Dirac distribution function,

$$
n_{F}\left(\omega_{f}\right)=\frac{1}{1+e^{\beta \omega_{f}}},
$$

where

$$
\omega_{f}=\left(p_{z}^{2}+M^{2}+2 k\left|q_{f}\right| B\right)^{1 / 2} .
$$

Notice that, contrary to the vacuum case, in the presence of a magnetic field the $u$ and $d$ condensates are different due to the different $u$ and $d$ electric charges, but the constituent masses of the $u$ and $d$ quarks are still equal; i.e., $M$ stands for both $M_{u}$ and $M_{d}$.

As mentioned earlier, the NJL model in the quasiparticle approximation is unable to describe inverse magnetic catalysis (IMC) [30,31], unless one imposes that the coupling constant $G$ of the model is $T$ - and $B$-dependent 43]]. A precise description of the lattice results for the $u$ and $d$ quark condensates is obtained within the NJL model with the parametrization [44],

$G(e B, T)=c(e B)\left[1-\frac{1}{1+e^{\beta(e B)\left[T_{a}(e B)-T\right]}}\right]+s(e B)$,

where $c(e B), \beta(e B), T_{a}(e B)$ and $s(e B)$ depend only on the magnitude of the external magnetic field. Their values for selected values of $B$ are given in Table 1 of Ref. [44]. All numerical results presented here refer to this parametrization.

The model just described was used to study the effects of a magnetic field on neutral pions in Refs. $[62,63]$, with results that agree with corresponding lattice QCD results [64]. Further evidence for the association of the IMC phenomenon with a magnetic field dependence decreasing the coupling strengths of quark-matter effective degrees of freedom was given in Refs. $[65,66]$.

\section{B. Electrical conductivity in presence of magnetic field: Classical and quantum}

Here we derive the electrical conductivity of magnetized hot quark matter (composed by the constituent quarks and antiquarks of flavor $u$ and $d$ ) employing the relaxation time approximation-closely following the approach of Ref. [23]. Initially we consider the classical description, ignoring the quantization of phase space in terms of Landau levels.

Let us consider a relativistic electrically charged fluid of constituent quarks (and antiquarks) with masses $M_{f}$ and energies $\omega_{f}$ [defined in Eq. (8)], characterized by distribution functions $f\left(\omega_{f}\right)$ described by the Boltzmann equation. In the electric-charge-transport picture, an external electric field is responsible for driving the system out of equilibrium. Let $f_{0}\left(\omega_{f}\right)=n_{F}\left(\omega_{f}\right)$ denote the equilibrium distribution, where $n_{F}\left(\omega_{f}\right)$ is the Fermi-Dirac distribution given in Eq. (7), and $f\left(\omega_{f}\right)=f_{0}\left(\omega_{f}\right)+\delta f\left(\omega_{f}\right)$ the distribution in the presence the electric force. The electric 
current $\boldsymbol{J}_{f}$ associated with a given quark flavor $f$ is expressed in terms of the quark distribution function by

$$
\begin{aligned}
\boldsymbol{J}_{f} & =\boldsymbol{J}_{0 f}+\boldsymbol{J}_{\delta f} \\
& =q_{f} g \int \frac{d^{3} p}{(2 \pi)^{3}} \boldsymbol{v}_{f}\left[f_{0}\left(\omega_{f}\right)+\delta f\left(\omega_{f}\right)\right],
\end{aligned}
$$

where $q_{f}$ is the charge of the quark (or antiquark), $g$ a spincolor degeneracy factor, and $\boldsymbol{v}_{f}=\boldsymbol{p} / \omega_{f}$ the quark velocity. The electrical conductivity of the fluid is a tensor relating the induced electric current and the electric field. Since we are working in the isospin-symmetric limit, we can drop the flavor index $f$ and concentrate on the contribution of a given quark-flavor $f$ to the conductivity and add up the contributions of each flavor at the end; the only difference comes from the electric charge $q_{f}$. From Ohm's law we have

$$
J_{\delta}^{i}=\sigma^{i j} E^{j} .
$$

The Boltzmann equation for the distribution function $f(\omega)$ of a quark of a given flavor under the influence of a generic external force $\boldsymbol{F}$ is given by

$$
\frac{\partial f}{\partial t}+\boldsymbol{v} \cdot \frac{\partial f}{\partial \boldsymbol{x}}+\boldsymbol{F} \cdot \frac{\partial f}{\partial \boldsymbol{p}}=\left(\frac{\partial f}{\partial t}\right)_{\mathrm{coll}},
$$

where $(\partial f / \partial t)_{\text {coll }}$ takes into account the collisional effects. For an uniformly distributed system, $\partial f / \partial \boldsymbol{x}=0$. The force $\boldsymbol{F}$ drives the system out of equilibrium and when the force is removed, scattering events described by $(\partial f / \partial t)_{\text {coll }}$ will restore equilibrium. The relaxation time approximation (RTA) consists in assuming for the collisional term,

$$
\left(\frac{\partial f}{\partial t}\right)_{\text {coll }}=-\frac{\delta f}{\tau_{c}}
$$

where $\tau_{c}$ is the relaxation time, the time required for the system to return to equilibrium after removing $\boldsymbol{F} .{ }^{1}$ The determination of the $T$ and $B$ dependence of $\tau_{c}$ is out of the scope of the present paper; when presenting results in the next section, we use values for $\tau_{c}$ in the range $0.2-10 \mathrm{fm} / \mathrm{c}$, the latter number being approximately the lifetime of the medium produced at the RHIC and the LHC. To assess the impact of a $T$ and $B$ dependence of $\tau_{c}$ on the results, we use a $\tau_{c}$ computed along the lines of the approach introduced in Ref. [50], based on quarkquark scattering cross-sections calculated with the contact

\footnotetext{
${ }^{1}$ Indeed, for $\boldsymbol{F}=0$ the solution of Eq. (12) in the RTA is
} given by

$$
f(t)=f_{0}+\left[f\left(t_{0}\right)-f_{0}\right] e^{-\left(t-t_{0}\right) / \tau_{c}},
$$

where $t_{0}$ is the time at which $\boldsymbol{F}$ is removed. interactions of the NJL model. The validity of the calculation is, however, restricted to $B \ll 10 m_{\pi}^{2}$, and therefore we present results for low values of $B$ only.

In the present context, $\boldsymbol{F}$ is the Lorentz's force, $\boldsymbol{F}=q(\boldsymbol{E}+\boldsymbol{v} \times \boldsymbol{B})$, where $q$ is the electric charge (recall that we are suppressing the flavor index $f$ ). It is useful to discuss first the situation with $\boldsymbol{B}=0$; the force is then $\boldsymbol{F}=q \boldsymbol{E}$. Assuming $\delta f \ll f_{0}$, Eq. (12) implies

$$
\begin{aligned}
\delta f & =-\tau_{c} q \boldsymbol{E} \cdot \frac{\partial f_{0}(\omega)}{\partial \boldsymbol{p}} \\
& =\tau_{c} \beta q \frac{\boldsymbol{p} \cdot \boldsymbol{E}}{\omega} f_{0}(\omega)\left[1-f_{0}(\omega)\right],
\end{aligned}
$$

where $\beta=1 / T$. Using this into Eq. (11), adding the contributions from quarks and antiquarks of flavor $u$ and $d$, taking into account that the spin-color degeneracy factor is $g=2 \times 3$, and the sum over flavor gives $\sum_{f} q_{f}^{2}=5 e^{2} / 9$, for the conductivity,

$$
\sigma^{i j}=\delta^{i j} \sigma
$$

with

$$
\sigma=e^{2} \beta \frac{20}{9} \int \frac{d^{3} p}{(2 \pi)^{3}} \frac{p^{2}}{\omega^{2}} \tau_{c} f_{0}(\omega)\left[1-f_{0}(\omega)\right]
$$

Next, we proceed with the derivation of $\sigma^{i j}$ in presence of a magnetic field $\boldsymbol{B}$ of arbitrary strength. In this situation, it is necessary to include the $\delta f$ contribution in the drift term in Eq. (12); otherwise, the term $\boldsymbol{v} \times \boldsymbol{B}$ in the Lorentz gives null contribution, because

$$
(\boldsymbol{v} \times \boldsymbol{B}) \cdot \frac{\partial f_{0}(\omega)}{\partial \boldsymbol{p}}=\left(\frac{\boldsymbol{p}}{\omega} \times \boldsymbol{B}\right) \cdot \boldsymbol{p} \frac{\partial f_{0}(\omega)}{\partial \omega}=0 .
$$

Including the $\delta f$ contribution in the drift term in Eq. (12), one obtains

$$
\delta f=-\tau_{c} q\left[\frac{1}{\omega} \boldsymbol{p} \cdot \boldsymbol{E} \frac{\partial f_{0}}{\partial \omega}+\left(\frac{\boldsymbol{p}}{\omega} \times \boldsymbol{B}\right) \cdot \frac{\partial(\delta f)}{\partial \boldsymbol{p}}\right] .
$$

Since any term proportional to $\boldsymbol{p}$ from $\partial(\delta f) / \partial \boldsymbol{p}$ leads to a vanishing contribution, one must have $\delta f \sim \boldsymbol{p} \cdot \mathcal{F}$ times a function of $\boldsymbol{p}^{2}$, where $\mathcal{F}$ is a vector that depends on the $\boldsymbol{E}$ and $\boldsymbol{B}$ fields. As shown in Ref. [23], Eq. (19) can be solved by writing $\delta f=-\boldsymbol{p} \cdot \mathcal{F} \partial f_{0} / \partial \omega$, with $\mathcal{F}=\alpha \boldsymbol{e}+\beta \boldsymbol{b}+$ $\gamma \boldsymbol{e} \times \boldsymbol{b}$, where $\boldsymbol{e}=\boldsymbol{E} / E$ and $\boldsymbol{b}=\boldsymbol{B} / B$, where $E=|\boldsymbol{E}|$ and $B=|\boldsymbol{B}|$. Specifically,

$$
-\boldsymbol{p} \cdot \mathcal{F}=-\tau_{c} q\left[\frac{1}{\omega} \boldsymbol{p} \cdot \boldsymbol{E}-\frac{1}{\omega} \boldsymbol{p} \cdot(\boldsymbol{B} \times \mathcal{F})\right],
$$

from which one obtains 


$$
\begin{gathered}
\alpha=q\left(\frac{\tau_{c}}{\omega}\right) \frac{1}{1+\left(\tau_{c} / \tau_{B}\right)^{2}} E, \\
\beta=q\left(\frac{\tau_{c}}{\omega}\right) \frac{\left(\tau_{c} / \tau_{B}\right)^{2}}{1+\left(\tau_{c} / \tau_{B}\right)^{2}}(\boldsymbol{e} \cdot \boldsymbol{b}) E, \\
\gamma=\left(\tau_{c} / \tau_{B}\right) \alpha=q\left(\frac{\tau_{c}}{\omega}\right) \frac{\left(\tau_{c} / \tau_{B}\right)}{1+\left(\tau_{c} / \tau_{B}\right)^{2}} E,
\end{gathered}
$$

where $\tau_{B}=\omega / q B$ is the inverse of the cyclotron frequency. Therefore, one obtains for $\delta f$,

$$
\begin{aligned}
\delta f= & -q\left(\frac{\tau_{c}}{\omega}\right) \frac{1}{1+\left(\tau_{c} / \tau_{B}\right)^{2}}\left[\delta_{i j}+\left(\tau_{c} / \tau_{B}\right) \epsilon^{i j k} b^{k}\right. \\
& \left.+\left(\tau_{c} / \tau_{B}\right)^{2} b^{i} b^{j}\right] \frac{\partial f_{0}(\omega)}{\partial \omega} p^{i} E^{j} .
\end{aligned}
$$

From Ohm's law in Eq. (11) and taking flavor summation of each quark or antiquark, having spin and color degeneracy factor $g=2 \times 3$, one immediately obtains for $\sigma^{i j}$,

$$
\sigma^{i j}=\delta^{i j} \sigma_{0}+\epsilon^{i j k} b^{k} \sigma_{1}+b^{i} b^{j} \sigma_{2},
$$

where $\sigma_{n}, n=0,1,2$, are given by

$\sigma_{n}=2 \beta \sum_{f=u, d} q_{f}^{2} \int \frac{d^{3} p}{(2 \pi)^{3}} \frac{p^{2}}{\omega^{2}} \frac{\tau_{c}\left(\tau_{c} / \tau_{B, f}\right)^{n}}{1+\left(\tau_{c} / \tau_{B, f}\right)^{2}} f_{0}(\omega)\left[1-f_{0}(\omega)\right]$

$=2 \beta \sum_{f=u, d} q_{f}^{2} \int \frac{d^{3} p}{(2 \pi)^{3}} \frac{p^{2}}{\omega^{2}} \frac{\tau_{c}\left(\tau_{B, f} / \tau_{c}\right)^{2-n}}{1+\left(\tau_{B, f} / \tau_{c}\right)^{2}} f_{0}(\omega)\left[1-f_{0}(\omega)\right]$,

where flavor index is marked in charge $\left(q_{f}\right)$ as well as in cyclotron time period $\left(\tau_{B, f}\right)$. These expressions are valid for quarks and antiquarks. Since $\sigma_{0}$ and $\sigma_{2}$ are proportional to the square of the electric charge $q_{f}$ and the medium we are considering contains equal numbers of quarks and antiquarks (zero chemical potential, $\mu=0$ ), the total $\sigma_{0}$ and $\sigma_{2}$ of the plasma is obtained by adding the quark and antiquark contributions. It means that additionally factor 2 will be multiplied in $\sigma_{0}$ and $\sigma_{2}$ of Eq. (27). This will not hold for $\sigma_{1}$. The quark and antiquark contributions to $\sigma_{1}$ have opposite sign due to the odd-function dependence of $\tau_{B}=$ $\omega /\left(q_{f} B\right)$ or $q_{f}$; therefore, the total Hall conductivity of the plasma is zero.

When $\boldsymbol{B}$ is in the $z$ direction, which implies $\hat{b}^{z}=1$ and $\hat{b}^{x}=\hat{b}^{y}=0$, the conductivity matrix elements are given by: $\sigma^{z z}=\sigma_{0}+\sigma_{2}=\sigma$ (longitudinal conductivity), $\sigma^{x x}=$ $\sigma^{y y}=\sigma_{0}$ (transverse conductivity), and $\sigma^{x y}=-\sigma^{y x}=\sigma_{1}$ (Hall conductivity), with the other components being zero. The $\sigma^{x y}=-\sigma^{x y}=\sigma_{1}$ components are absent in the zero magnetic field case, Eq. (17). This reveals that the spaceanisotropic nature of the conductivity which, according to Eqs. (26) and (27), is controlled by the ratio between the two timescales $\tau_{c}$ and $\tau_{B}$. This anisotropy shows interesting features for weak and strong magnetic fields.

The two-way expression of the $\sigma_{n}$ components in Eqs. (26) and (27) with respect to the $\tau_{c}$ and $\tau_{B}$ dependence is useful for discussing the weak- and strong-field limits of the $\sigma_{n}$. For a weak magnetic field, $\tau_{c} / \tau_{B}$ is small. Expanding the integrand Eq. (26) up to second order in $\tau_{c} / \tau_{B}$ one obtains

$$
\begin{aligned}
\left(\begin{array}{c}
\sigma_{0} \\
\sigma_{1} \\
\sigma_{2}
\end{array}\right) \simeq & 2 \beta \sum_{f=u, d} q_{f}^{2} \int \frac{d^{3} p}{(2 \pi)^{3}} \frac{\boldsymbol{p}^{2}}{\omega^{2}} \tau_{c}\left(\begin{array}{c}
1-\left(\tau_{c} / \tau_{B, f}\right)^{2} \\
\tau_{c} / \tau_{B, f} \\
\left(\tau_{c} / \tau_{B, f}\right)^{2}
\end{array}\right) \\
& \times f_{0}(\omega)\left[1-f_{0}(\omega)\right] .
\end{aligned}
$$

This shows that a small space-anisotropic conduction is already present in the weak-field limit. The $\sigma^{x x}$ and $\sigma^{y y}$ diagonal elements are reduced from the zero-field case, whereas the $\sigma^{z z}=\sigma_{0}+\sigma_{2}=\sigma$ component is unchanged. For a strong magnetic field, $\tau_{c} / \tau_{B}$ is large, and Eq. (27) up to second order in $\tau_{B} / \tau_{c}$ can be written as

$\sigma_{n} \simeq 2 \beta \sum_{f=u, d} q_{f}^{2} \int \frac{d^{3} p}{(2 \pi)^{3}} \frac{\boldsymbol{p}^{2}}{\omega^{2}} \tau_{c}\left(\tau_{B, f} / \tau_{c}\right)^{2-n} f_{0}(\omega)\left[1-f_{0}(\omega)\right]$.

One sees that as $B \rightarrow \infty$, all the components of the conductivity tensor, with the exception of $\sigma^{z z}$, vanish.

Up to now, classical physics was used to determine the conductivity. As seen in Sec. II A, a proper quantum mechanical treatment of electric charge dynamics in a magnetic field leads to one-dimensional dynamics and energy quantization in terms of Landau levels. In practical terms, these effects amount to modifying the results above by the following replacements:

$\omega=\left(\boldsymbol{p}^{2}+M^{2}\right)^{1 / 2} \rightarrow \omega_{f, k}=\left(p_{z}^{2}+M^{2}+2 k\left|q_{f}\right| B\right)^{1 / 2}$,

$3 \times 2 \int \frac{d^{3} p}{(2 \pi)^{3}} \frac{p^{2}}{3 \omega^{2}} \rightarrow 3 \sum_{k=0}^{\infty} \alpha_{k} \frac{|q| B}{2 \pi} \int_{-\infty}^{+\infty} \frac{d p_{z}}{2 \pi} \frac{p_{z}^{2}}{\omega^{2}}$,

where in the last line we used the fact that the lowest Landau level (LLL), $k=0$, is spin-polarized-recall that $\alpha_{k}=2-\delta_{k, 0}$. In the quantum picture energy will depend on Landau level $k$ and flavor $f$ both; therefore, it is marked as $\omega_{f, k}$. Hence, using those changes, the longitudinal conductivity of $u / d$ flavor quark/antiquark in the quantum description is given by 


$$
\sigma^{z z}=3 \beta \sum_{f=u, d} q_{f}^{2} \sum_{k=0}^{\infty} \alpha_{k} \frac{\left|q_{f}\right| B}{2 \pi} \int_{-\infty}^{+\infty} \frac{d p_{z}}{2 \pi} \frac{p_{z}^{2}}{\omega_{f, k}^{2}} \tau_{c} f_{0}\left(\omega_{f, k}\right)\left[1-f_{0}\left(\omega_{f, k}\right)\right] .
$$

Considering charges of $u$ and $d$ quarks and their antiparticle contribution, Eq. (32) will be modified to

$$
\sigma^{z z}=6 \beta \sum_{f=u, d} q_{f}^{2} \sum_{k=0}^{\infty} \alpha_{k} \frac{\left|q_{f}\right| B}{2 \pi} \int_{-\infty}^{+\infty} \frac{d p_{z}}{2 \pi} \frac{p_{z}^{2}}{\omega_{f, k}^{2}} \tau_{c} f_{0}\left(\omega_{f, k}\right)\left[1-f_{0}\left(\omega_{f, k}\right)\right] .
$$

The transition between the classical and quantum regimes in $\sigma^{z z}$ can be obtained by varying $\tau_{c}$ for a fixed $\tau_{B}$. This analysis is made in the next section.

In the strong field limit, the dominant contribution to $\sigma^{z z}$ comes from the lowest Landau level (LLL). At LLL, energy expression will be transformed as

$$
\omega_{f, k=0}=\omega_{0}=\sqrt{p_{z}^{2}+M^{2}},
$$

and flavor summation can be done as

$$
\begin{aligned}
\sigma^{z z} & =2 \times 3\left[\left(\frac{2}{3}\right)^{3}+\left(\frac{1}{3}\right)^{3}\right] e^{2} \beta \sum_{k=0}^{\infty} \alpha_{k} \frac{|e| B}{2 \pi} \int_{-\infty}^{+\infty} \frac{d p_{z}}{2 \pi} \frac{p_{z}^{2}}{\omega_{0}^{2}} \tau_{c} f_{0}\left(\omega_{0}\right)\left[1-f_{0}\left(\omega_{0}\right)\right] \\
& =e^{2} \beta \frac{|e| B}{\pi} 2 \int_{0}^{\infty} \frac{d p_{z}}{2 \pi} \frac{p_{z}^{2}}{\omega_{0}^{2}} \tau_{c} f_{0}\left(\omega_{0}\right)\left[1-f_{0}\left(\omega_{0}\right)\right] \\
& =e^{2} \beta \frac{|e| B}{\pi^{2}} \int_{M}^{\infty} d \omega_{0} \frac{\sqrt{\omega_{0}^{2}-M^{2}}}{\omega_{0}} \tau_{c} f_{0}\left(\omega_{0}\right)\left[1-f_{0}\left(\omega_{0}\right)\right] .
\end{aligned}
$$

One can check that Eq. (35) coincides with Eq. (D.5) of Ref. [21]. Equation (33) contains quantum effects leading to Landau-level quantization but does not contain contributions due to the quantum chiral anomaly. The latter causes an imbalance between left- and right-handed quark and antiquark currents and can affect the longitudinal electrical conductivity, with the effect increasing with $B$ [67]. Such an effect on the $T$ and $B$ dependence on the conductivity has not yet been considered in the present context, and we reserve examination of such effects for a future publication.

\section{NUMERICAL RESULTS AND DISCUSSION}

First, we present results for the constituent quark mass $M$ as a function of the temperature $T$ and the magnetic field $B$. The quark mass is a key ingredient in the calculation of the electrical conductivity; it enters in the definition of quasiparticle energy $\omega_{f}$, which in turn enters in the definition of cyclotron frequency $\tau_{B}$, and in the Fermi-Dirac distribution $n_{F}\left(\omega_{f}\right)$-it is the link between dynamical chiral symmetry breaking and transport properties. Next, we present results for the conductivity using temperature and magnetic field independent values of the relaxation time. We start presenting results for the conductivity components and discuss how its anisotropy is affected by $T$ and $B$. We examine the impact of phase-space quantization on the longitudinal component of the conductivity and examine the validity of truncating phase space to the lowest Landau level (LLL). We also discuss how the phenomenon of the inverse magnetic catalysis affects the electrical conductivity. We finalize our study examining the impact of using a $T$ and $B$ dependent $\tau_{c}$ on the longitudinal conductivity.

The constituent quark mass $M=M(T, B)$ was obtained by solving the gap equation, i.e., Eq. (2) for different values of $T$ and $B$. As mentioned earlier, when $m_{u}=m_{d}=m$, the equality $M_{u}=M_{d}=M$ holds both in vacuum and when $B \neq 0$, but the equality $\langle\bar{u} u\rangle=\langle\bar{d} d\rangle$ holds only in vacuum. The parameters of the model are the current quark mass $m$, the coupling $G$ and the cutoff $\Lambda$. Since the interest is to take into account the IMC phenomenon in the phenomenology of the electrical conductivity, those parameters were fitted to reproduce the lattice data of Ref. [31]. A precise description of the lattice data for the quark condensate is obtained in [44] when using the parametrization given in Eq. (9). The values of the parameters $c(B), \beta(B), T_{a}(B)$, and $s(B)$ defining the fitting formula in Eq. (9) are displayed in Table 1 of Ref. [44]. The value of the coupling that reproduces the $T=B=0$ quark condensates of lattice data of Ref. [31] is $G=4.50 \mathrm{GeV}^{-2}$. The values of the remaining parameters are $m=5.5 \mathrm{MeV}$ and $\Lambda=650 \mathrm{MeV}$, and they correspond to the vacuum pion decay constant, $f_{\pi}=93 \mathrm{MeV}$, and vacuum pion mass, $m_{\pi}=140 \mathrm{MeV}$. In the numerical results for the fixed coupling constant we have 


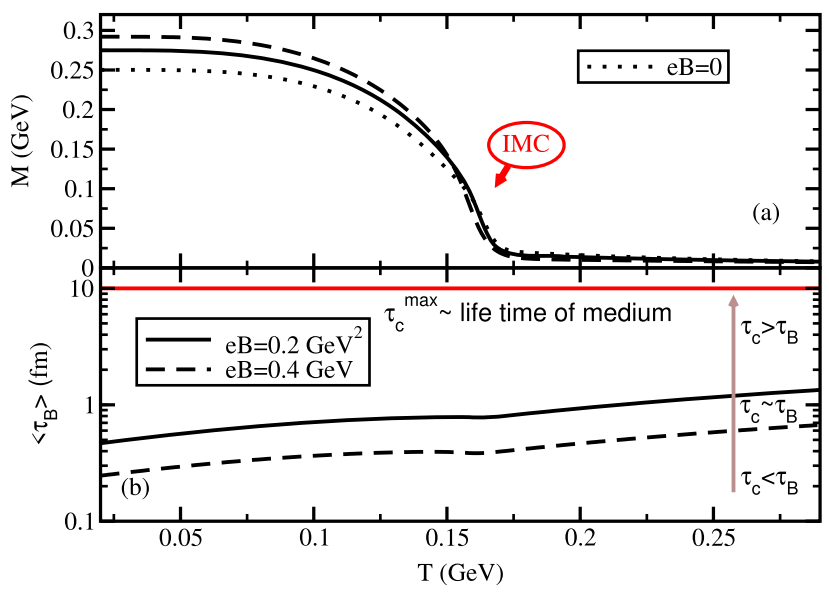

FIG. 1. Temperature dependence of (a) constituent quark mass $M$ and (b) momentum average of the cyclotron time period $\left\langle\tau_{B}\right\rangle$ [defined in Eq. (36)] for different values of the magnetic field.

used $G(0,0)=4.6311 \mathrm{GeV}^{-2}$; i.e., the value obtained in Eq. (9) that was fitted to the lattice at high temperature region (more details can be found in [44]).

Figure 1(a) displays the constituent quark mass $M$ as a function of the temperature for zero and nonzero magnetic field. For small values of $T$, the mass increases with $B$, whereas for temperatures near the pseudocritical temperature $T_{\mathrm{pc}} \simeq 0.17 \mathrm{GeV}$ it decreases with $B$. These features, which are carried over to the $u$ and $d$ condensates, characterize the phenomena of magnetic catalysis (MC) at low $T$ and inverse magnetic catalysis (IMC) at $T \sim T_{\mathrm{pc}}$. The impact of these features on the electrical conductivity, in particular on its anisotropy, will be discussed shortly.

The anisotropy is controlled by the ratio of two timescales, the relaxation time $\tau_{c}$ and the cyclotron time $\tau_{B}=\omega / e B$. As mentioned earlier, it is out of our scope to determine $\tau_{c}$ from microscopic dynamics. We recall that $\tau_{c}$ depends on parameters of the heavy-ion collision as the centrality of the collision (impact parameter) and the energy and size of the colliding nuclei. Here we use values for $\tau_{c}$ in the range $0.1 \mathrm{fm} / \mathrm{c}<\tau_{c}<5 \mathrm{fm} / \mathrm{c}$ and consider as upper bound the value $\tau_{c}=10 \mathrm{fm} / \mathrm{c}$, which is approximately the lifetime of the medium produced at the RHIC and LHC. The cyclotron time $\tau_{B}=\omega / e B$ is a momentum dependent quantity. For a given momentum, $\tau_{B}$ decreases with $B$ and $T$; the decrease with $B$ comes mainly from the trivial $1 / e B$ factor, whereas the decrease in $T$ comes from $M$ in $\omega=\left(\boldsymbol{p}^{2}+M^{2}\right)^{1 / 2}$. But $\tau_{B}$ is integrated over a momentum range bounded by the thermal distribution $f_{0}(\omega)$. To assess the effect of this integration, we have defined the following average:

$$
\left\langle\tau_{B}\right\rangle=\frac{\int d^{3} \boldsymbol{p} \tau_{B} f_{0}(\omega)}{\int d^{3} \boldsymbol{p} f_{0}(\omega)}=\frac{1}{\int d^{3} \boldsymbol{p} f_{0}(\omega)} \int d^{3} \boldsymbol{p} \frac{\omega}{e B} f_{0}(\omega),
$$

with $\omega=\left(\boldsymbol{p}^{2}+M^{2}\right)^{1 / 2}$. The temperature dependence of $\tau_{B}(B, T)$ is depicted in Fig. 1(b). This average increases with $T$ despite the decrease of $M$ with $T$. This is because $f_{0}(\omega)$ dominates the temperature dependence of the integrand; although the numerator and denominator in Eq. (36) both grow with $T$, the numerator grows faster. This feature has important consequences for the temperature dependence of the anisotropy of the conductivity. The vertical arrow in Fig. 1(b) indicates the direction of growth of $\left\langle\tau_{B}\right\rangle$ with $B$ and identifies regions of weak $\left(\tau_{c}<\tau_{B}\right)$ and strong $\left(\tau_{c}>\tau_{B}\right)$ magnetic field.

Next, we present our results for the electrical conductivity-we recall the $B$ is taken in the $z$ direction. We start with the classical results. We recall that for zero magnetic field, the electrical conductivity tensor is
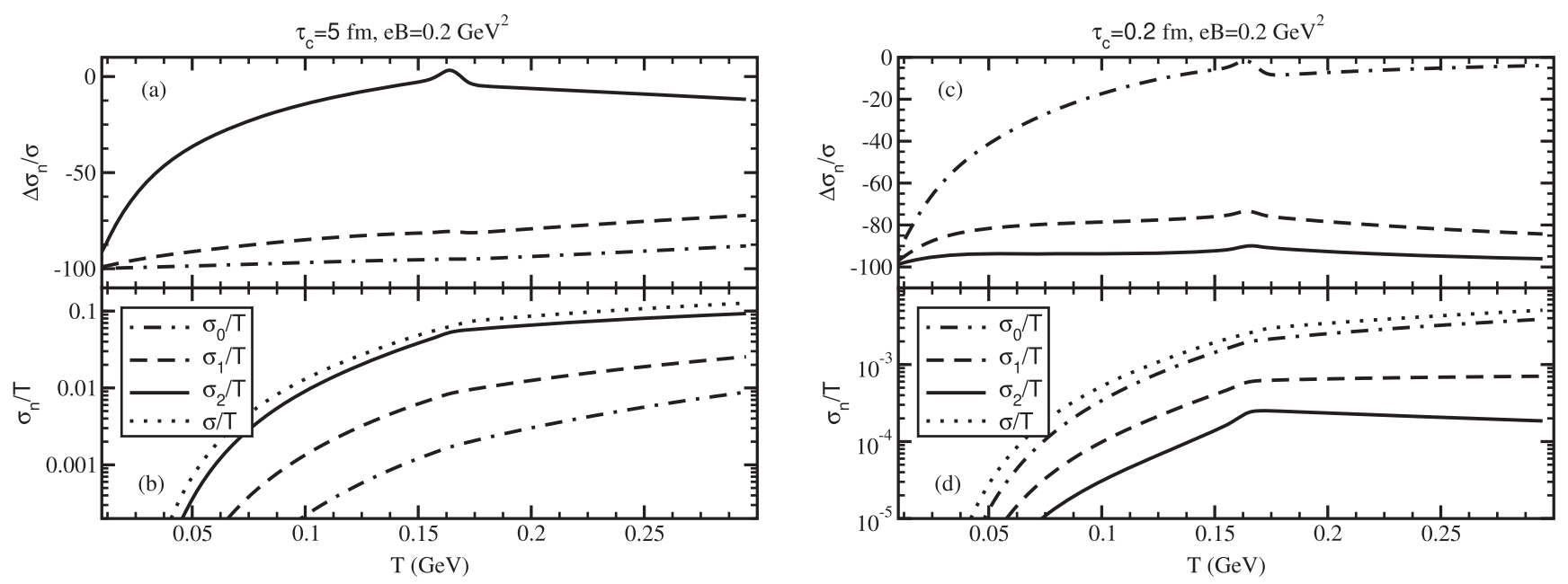

FIG. 2. Temperature dependence of the difference (\%) $\Delta \sigma_{n} / \sigma=\left(\sigma_{n}-\sigma\right) / \sigma$ and of $\sigma_{n} / T$, corresponding to the three components of electrical conductivities for $e B=0.2 \mathrm{GeV}^{2}$ and two values of the relaxation time, $\tau_{c}=5 \mathrm{fm} / \mathrm{c}$ (left) and $\tau_{c}=0.2 \mathrm{fm} / \mathrm{c}$. Here the classical expressions for the $\sigma_{n}$, Eq. (28), are used. 


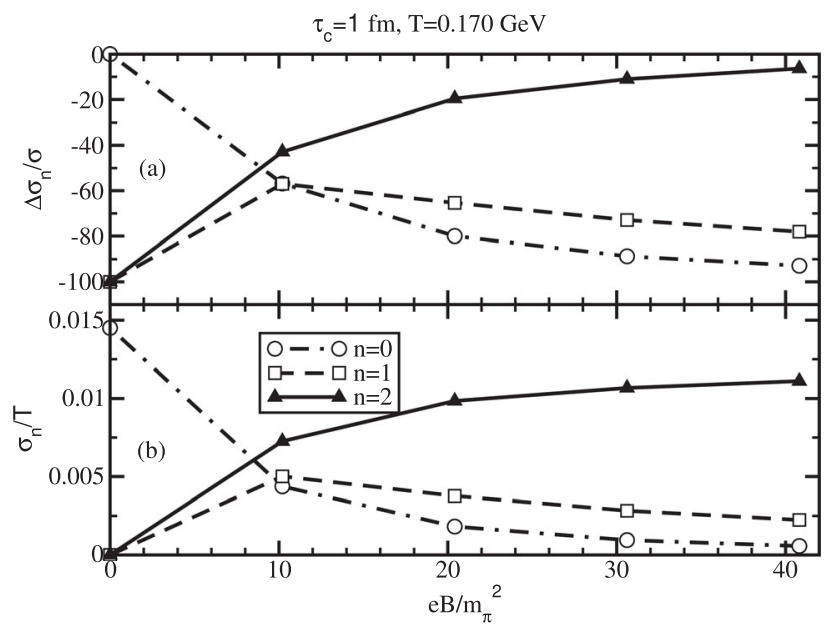

FIG. 3. Magnetic field dependence of the difference (\%) $\Delta \sigma_{n} / \sigma=\left(\sigma_{n}-\sigma\right) / \sigma$ and the three components of electrical conductivities for $T=0.170 \mathrm{GeV}$ and $\tau_{c}=1 \mathrm{fm} / \mathrm{c}$. Here the classical expressions for the $\sigma_{n}$, Eq. (28), are used.

diagonal, with all the diagonal elements being equal to $\sigma$, given by Eq. (17). When $B \neq 0$, there is anisotropy in the conductivity, as indicated by Eqs. (26) and (27). To quantify the anisotropy, we define the differences $\Delta \sigma_{n} / \sigma=\left(\sigma_{n}-\sigma\right) / \sigma$. In Fig. 2, we present results for the temperature dependence of $\Delta \sigma_{n} / \sigma$ and of $\sigma_{n} / T$. The results are for $e B=0.2 \mathrm{GeV}^{2}$ and two values for the relaxation time, $\tau_{c}=5 \mathrm{fm} / \mathrm{c}$ and $\tau_{c}=0.2 \mathrm{fm} / \mathrm{c}$. For this value of $B$ and within the temperature range shown in the figure, $\tau_{c}=5 \mathrm{fm} / \mathrm{c}$ characterizes a strong magnetic field whereas $\tau_{c}=0.2 \mathrm{fm} / \mathrm{c}$ characterizes a weak magnetic field. It can be seen from Fig. 2 that $\sigma_{n}$ increase with the temperature having a kink at $T \simeq T_{\mathrm{pc}}$, after which the rate of increase is substantially reduced. This kink turns into a strong peak when using a $T$ and $B$ dependent $\tau_{c}$, as

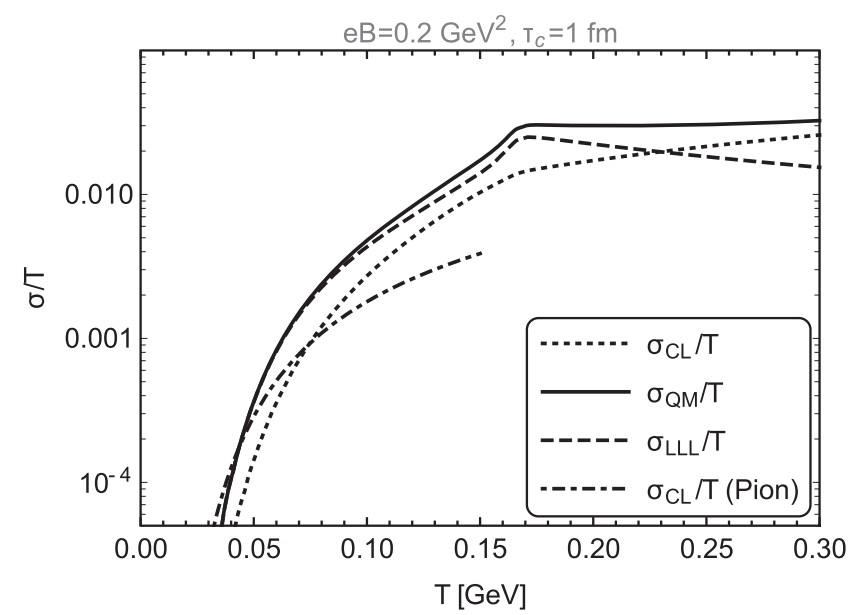

we discuss at the end of this section. We conclude that the electrical conductivity becomes almost independent of $T$ for $T>T_{\mathrm{pc}}$, when the constituent quark mass stops changing with $T$, since then $M \approx m$. In addition, $\sigma^{x x}=$ $\sigma^{y y}<\sigma^{z z}$ for $\tau_{c}=5 \mathrm{fm} / \mathrm{c}$ (strong field), whereas $\sigma^{x x}=$ $\sigma^{y y} \approx \sigma^{z z}$ for $\tau_{c}=0.2 \mathrm{fm} / \mathrm{c}$ (weak field), as expected.

Interesting insight is gained by examining the conductivity as a function of the magnetic field at the pseudocritical temperature. Figure 3 displays the $B$ dependence of $\Delta \sigma_{n} / \sigma$ and $\sigma_{n} / T$ at $T=T_{\mathrm{pc}}=0.170 \mathrm{GeV}$, for $\tau_{c}=1 \mathrm{fm} / \mathrm{c}$. The anisotropy is enhanced as $B$ increases.

The results discussed up to here used the classical expression for the conductivity, expected to provide a reasonable approximation for weak magnetic fields. The quantum description of charge transport leads to onedimensional dynamics and quantization of the density of states in terms of Landau levels in a plane perpendicular to the magnetic field. Taking the magnetic field in the $z$ direction, the longitudinal conductivity $\sigma^{z z}$ is then given by Eq. (33), instead of $\sigma^{z z}=\sigma_{0}+\sigma_{2}=\sigma$, with $\sigma$ given by Eq. (17). In the following we have compared the classical and quantum predictions of the model, and also examined the validity of truncating the density of states to the lowest Landau level (LLL).

The classical and quantum results for $\sigma^{z z}$ are shown in Fig. 4. In the left panel we plotted $\sigma^{z z}$ as function of $T$ for $e B=0.2 \mathrm{GeV}$ and on the right panel as a function of $B$ for $T=T_{\mathrm{pc}}=0.170 \mathrm{GeV}$. All results are for $\tau_{c}=1 \mathrm{fm} / \mathrm{c}$. The classical and quantum results disagree in the entire temperature range shown in the figure, but the difference between them gets smaller as the temperature increases, as it should. In addition, the LLL approximation is good only for temperatures below $0.1 \mathrm{GeV}$. Regarding the magnetic field dependence of $\sigma^{z z}$, the classical and quantum predictions are completely different; this is expected, as the

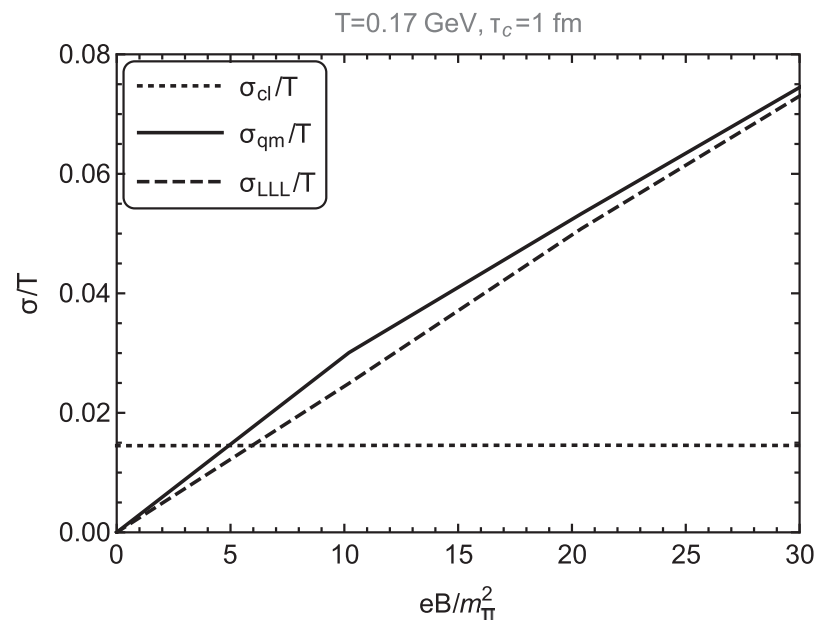

FIG. 4. Temperature (left panel) and magnetic field (right panel) dependent longitudinal electrical conductivity $\sigma^{z z}$. (For quark matter) dotted curve: classical, Eq. (27); solid curve: quantum, Eq. (33), using 100 Landau levels; dashed curve: LLL approximation. (For pion matter) dash-dotted curve: classical, Eq. (27). 
Lorentz force is ineffective along the direction of the magnetic field. The only effect of the magnetic field is via the mass $M$, but at $T=0.170 \mathrm{GeV}$, one already has $M \simeq m$. The truncation to the LLL is a poor approximation, particularly for values of $B$ in the region $6 m_{\pi}^{2}<e B<$ $10 m_{\pi}^{2}$. We find that the quantum curve in the right panel of Fig. 4 approximately proportional to $e B$ and crosses the horizontal classical curve around $e B=5 m_{\pi}^{2}$. So beyond the $e B=5 m_{\pi}^{2}$ (for $T=0.170 \mathrm{GeV}, \tau_{c}=1 \mathrm{fm}$ ), the quantum picture is revealing but within $e B=0-5 m_{\pi}^{2}$ the classical picture will be our matter of interest. Actually, due to numerical drawback we have not properly generated quantum results within $e B=0-5 m_{\pi}^{2}$, which is ultimately supposed to merge with classical results. In general we can roughly assume low $T$ and/or high $e B$ as quantum zone and remaining part as classical zone.

It is important to point out that our finding; i.e., the longitudinal conductivity $\left(\sigma^{z z}\right)$ increases with $B$ whereas the transverse conductivity $\left(\sigma_{x x}=\sigma_{y y}\right)$ decreases with $B$, is in qualitative agreement with the most recent lattice simulations of full QCD of Ref. [19] (this reference presents results for $T=0.2 \mathrm{GeV}$ and $T=0.25 \mathrm{GeV}$ ).

At low temperatures, the relevant degrees of freedom of the medium are hadrons. We make a comparison between the low temperature NJL results with those expected employing hadronic degrees of freedom; here we restrict the hadronic phase to pionic matter. Using the classical expression of longitudinal conductivity given in Eq. (29), we have obtained the conductivity for pionic matter. The required modifications in Eq. (29) are the transported electric charge and mass and the distribution function: $\sum q_{f}^{2}$ is replaced by $2 e^{2}$, and the $T$ and $B$ dependent quark mass is replaced by a constant $140 \mathrm{MeV}$ pion mass, and $f_{0}$ will be the Bose-Einstein distribution instead of FermiDirac distribution. The results are shown by dash-dotted line in the left panel of Fig. (4). We notice qualitatively similar temperature dependences of $\sigma_{z z}$ for both quark and pionic matter at low temperatures. We find that at $0<T<0.070 \mathrm{GeV}$, the pion component is lager than the quark component but an opposite trend is found at $0.070<T<0.170 \mathrm{GeV}$. To realize actual mapping of the NJL model with hadronic phase, we should go through their normalized value. From Fig. 4, one can notice that $\sigma / T=0.0018-0.0047$ for pion medium and $\sigma / T=$ 0.0028-0.014 for NJL based quark medium within $T=0.1-0.17 \mathrm{GeV}$. However, if we normalize their charge factors, then we will get the ranges,

$$
\begin{aligned}
& \frac{\sigma}{T}\left(\frac{1}{2 e^{2}}\right)=\frac{0.0009}{e^{2}}-\frac{0.0023}{e^{2}}, \quad \text { (for pion medium) } \\
& \frac{\sigma}{T}\left(\frac{9}{5 e^{2}}\right)=\frac{0.005}{e^{2}}-\frac{0.025}{e^{2}},
\end{aligned}
$$

(for NJL-based quark medium).
Now, we notice that by normalizing the charge factor, longitudinal conductivity of pion medium are lower than NJL-based quark medium in the entire hadronic temperature $0<T<0.170 \mathrm{GeV}$. If one follows the hadron resonance gas (HRG) model [54] and add higher mass resonances with a pion component, then the hadronic matter conductivity might be close to the conductivity of NJL-based quark medium. For thermodynamical quantities like pressure, energy density etc. the approximate equivalence of the NJL model, LQCD and HRG model in low temperature $(T=0.1-0.17 \mathrm{GeV})$ is quite a well-known fact.

Finally, we examine the significance of IMC on the electrical conductivity. Figure 5 shows two sets of results for the $T$ - and $B$-dependence of $\Delta \sigma_{n} / \sigma$ : one is calculated with a constituent quark mass $M$ with the $G(e B, T)$ coupling of Eq. (9), and the other is calculated with a $G$ fixed to $G=G(e B, T)=4.6311 \mathrm{GeV}^{-2}$. We recall that the NJL model describes IMC at $T \sim T_{\mathrm{pc}}=0.170 \mathrm{GeV}$ only when $G=G(e B, T)$; otherwise it gives magnetic catalysis (MC) in the entire range of temperatures shown in the figure. IMC produces a kink in the $\Delta \sigma_{n}$ at $T \simeq T_{\mathrm{pc}}$, whereas MC produces featureless $\Delta \sigma_{n}$. The physical origin of this kink structure can be understood by comparing the constituent quark mass $M$ calculated with a $G=G(e B, T)$ and with a $G$ fixed. The comparison is better made by defining the ratio $\Delta M / M_{0}$, where $\Delta M=M(B)-M_{0}$ and $M_{0}=M(B=0)$. Figure 6 displays the comparison: the kink is due to a rapid inversion of the temperature dependence of $M$ when $G=G(e B, T)$. In the following, we show that this kink in the conductivity becomes a prominent peak when using a $T$ and $B$ dependent $\tau_{c}$.

The results above are for fixed values of the relaxation time $\tau_{c}$. Next, we examine the impact of a $T$ and $B$ dependent $\tau_{c}$ on the longitudinal conductivity. A a $T$ and $B$ dependent $\tau_{c}$ can be calculated within the NJL model:

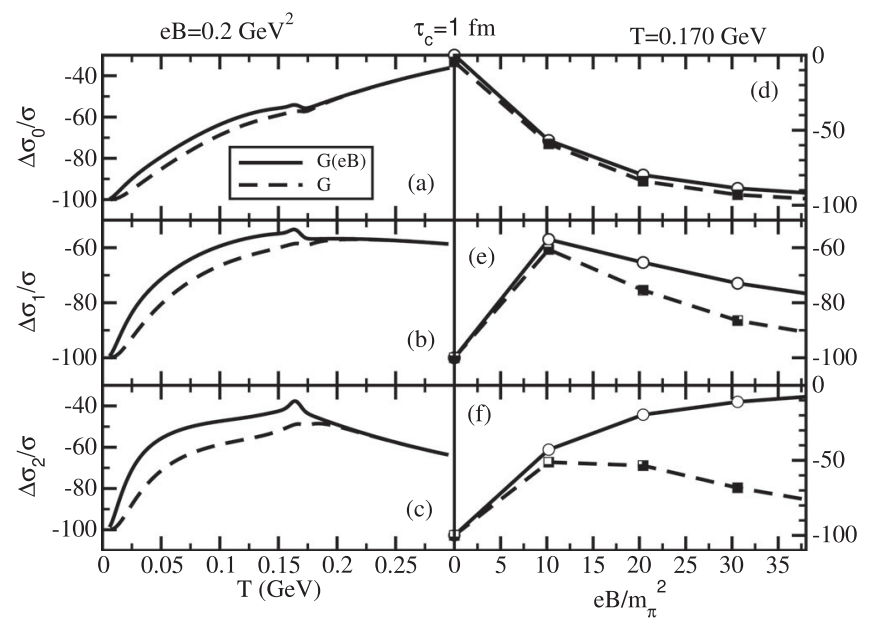

FIG. 5. Effect of inverse magnetic catalysis (IMC) on the electrical conductivity. The solid lines are obtained with a constituent quark mass $M$ calculated with the NJL coupling $G=G(e B, T)$ and the dashed lines with $G=G(0,0)=4.6311 \mathrm{GeV}^{-2}$. 


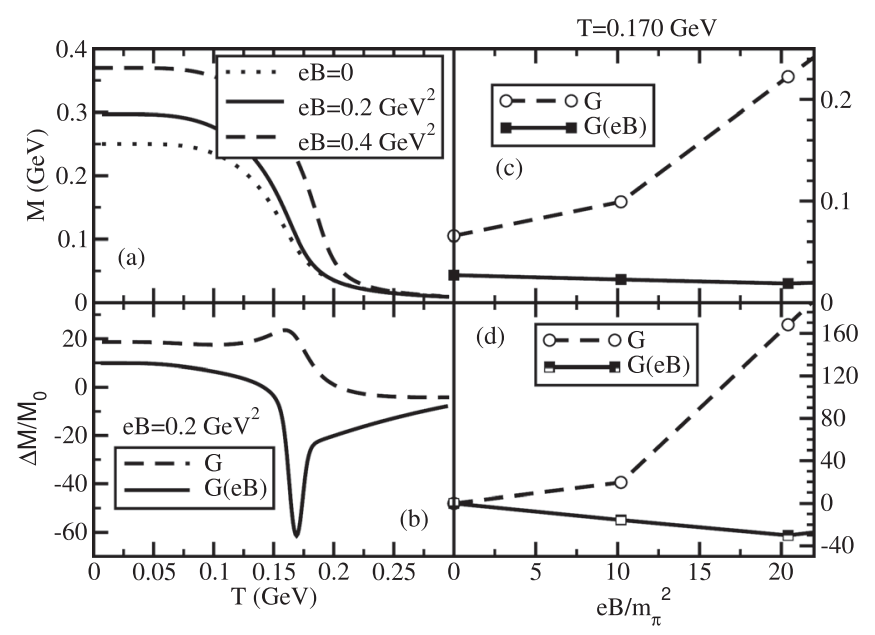

FIG. 6. (a): Constituent quark mass for fixed constant coupling $G=G(0,0)=4.6311 \mathrm{GeV}^{-2}$. (c) Constituent quark mass for $G=G(e B, T)$. (b) and (d): $\Delta M / M_{0}$, where $\Delta M=M(B)-M_{0}$ and $M_{0}=M(B=0)$.

for $B=0$, the temperature dependence of $\tau_{c}$ was computed in Refs. [68-71] using quark- $\pi$ and quark- $\sigma$ loops, in Refs. [72-76] it was computed using quark-quark scattering diagrams with $\pi$ and $\sigma$ exchanges, and in Ref. [77] it was computed using both quark-meson loops and quark-quark scatterings. The extension of those calculations to $B \neq 0$ is technically difficult, but it can be simplified employing quark-quark scattering processes from the contact interactions of the NJL model [50]. Here we follow this approach using a fixed $G$ and a $G(T, e B)$ coupling as above.

The relaxation time is given by [50]

$$
\begin{aligned}
\frac{1}{\tau_{c}(T, e B)} & =\Gamma_{c}(T, e B) \\
& =\frac{\sum_{k=0}^{\infty} \alpha_{k} \frac{\left|q_{f}\right| B}{2 \pi} \int \frac{d p_{a}^{z}}{2 \pi} \Gamma\left(T, e B, \boldsymbol{p}_{a}\right) f_{0}\left(\omega_{a}\right)}{\sum_{k=0}^{\infty} \alpha_{k} \frac{\left|q_{f}\right| B}{2 \pi} \int \frac{d p_{a}^{z}}{2 \pi} f_{0}\left(\omega_{a}\right)},
\end{aligned}
$$

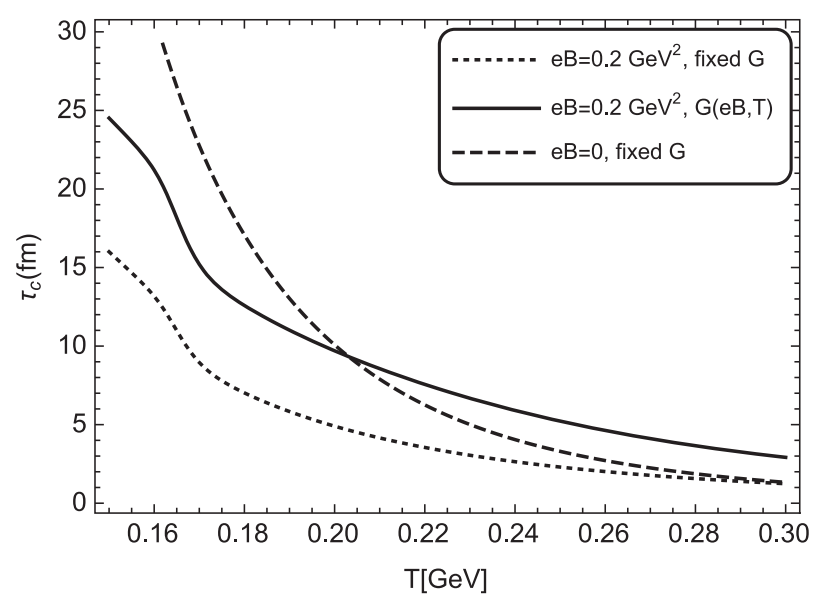

where $\omega_{a}$ is given in Eq. (8), $\Gamma(T, e B)$ is the momentum average of the collisional width (collisional frequency) of probe particle with momentum $\boldsymbol{p}_{a}, \Gamma\left(T, e B, \boldsymbol{p}_{a}\right)$. The collisional width $\Gamma\left(T, e B, \boldsymbol{p}_{a}\right)$ is given in terms of quarkquark scattering cross sections as

$$
\begin{aligned}
\Gamma_{c}\left(T, e B, \boldsymbol{p}_{a}\right)= & \frac{1}{\tau_{c}\left(T, e B, \boldsymbol{p}_{a}\right)} \\
= & \sum_{b} \sum_{k=0}^{\infty} \alpha_{k} \frac{\left|q_{f}\right| B}{2 \pi} \int \frac{d p_{b z}}{2 \pi} \sigma_{a b}\left(T, e B, \boldsymbol{p}_{a}, \boldsymbol{p}_{b}\right) \\
& \times v_{a b}\left(T, e B, \boldsymbol{p}_{a}, \boldsymbol{p}_{b}\right) f_{0}\left(\omega_{b}\right)
\end{aligned}
$$

where

$$
v_{a b}\left(T, e B, \boldsymbol{p}_{a}, \boldsymbol{p}_{b}\right)=\frac{\left\{\left(\omega_{a}+\omega_{b}\right)^{2}-4 M_{Q}^{2}(T)\right\}^{1 / 2}\left(\omega_{a}+\omega_{b}\right)}{2 \omega_{a} \omega_{b}},
$$

is relative velocity. The $\sigma_{a b}$ cross sections are obtained [50] using the standard quantum field theoretical relation of $2 \rightarrow 2$ scattering as

$\sigma_{a b}=\frac{1}{16 \pi\left(\omega_{a}+\omega_{b}\right)^{2}} \overline{|M|_{a b}^{2}}=\frac{\left(\omega_{a}+\omega_{b}\right)^{2}}{16 \pi} G^{2}(e B, T)$.

Hence, we have $T$ and $B$ dependence in the cross sections through the $\omega_{a}$ and $\omega_{b}$, and also through the coupling $G(e B, T)$. Figure 7(a) shows the temperature dependence of $\tau_{c}$ for different values of $B$, for $G$ fixed and $G(e B, T)$. Figure 7(b) shows the corresponding temperature dependence of longitudinal conductivity. For numerical simplicity, both of these plots are for the lowest Landau level. The interpretation of the results is straightforward, as we discuss next.

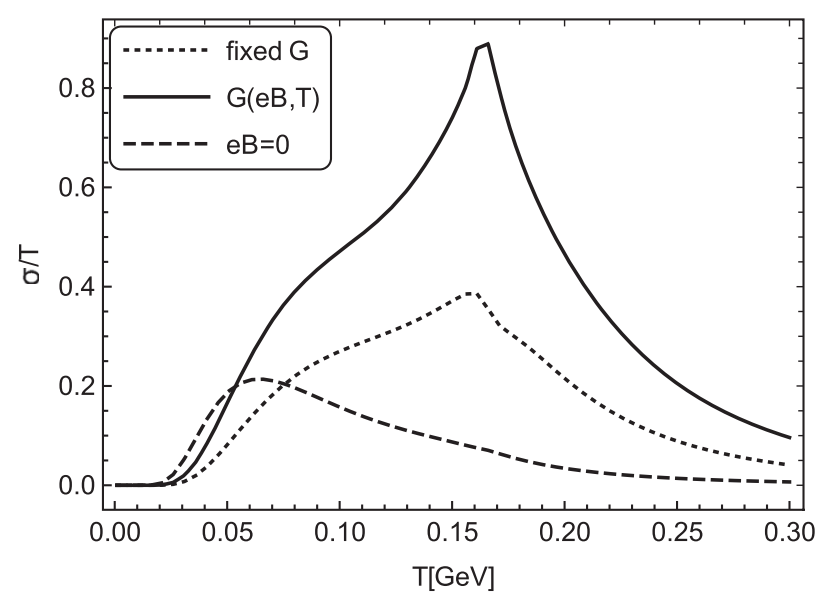

FIG. 7. Temperature dependence of $\tau_{c}$ (left panel) and $\sigma / T$ (right panel) for different values of $e B$ and fixed (dashed lines) and $T$ and $B$ dependent (solid and dotted lines) NJL coupling constants. 
Qualitatively, Eq. (39) means $\tau_{c}^{-1}=\sigma_{a b} \times v_{a b} \times \rho$, where $\rho$ is number density. Being inversely proportional to $\rho, \tau_{c}$ decreases with the increase of $T$. For massless quarks, a Stefan-Boltzmann type density follows a $T^{3}-$ dependence, which is definitely modified for a $T$-dependent constituent quark mass. Now, for a nonzero magnetic field, the phase-space distribution $f_{0}(\omega)$ in general is enhanced, as seen in the quark condensate and constituent quark mass. This means that $\tau_{c}$ is reduced due to the magnetic field. This reduction of $\tau_{c}$ can be seen in Fig. 7(a) by comparing the dashed and dotted lines. We note that these two curves merge at high $T$, when the quark condensate becomes very small. These results are for a fixed value of the coupling $G$, the model describes magnetic catalysis only. The effect of inverse magnetic catalysis on $\tau_{c}$, obtained with a $G(e B, T)$ coupling constant, is shown by the solid line in Fig. 7(a): a rapid fall of $\tau_{c}$ at $T_{\mathrm{pc}}$. In addition, there is an inversion of the temperature dependence at low (high) $T, \tau_{c}$ is smaller (larger) than the corresponding values at $B=0$. This rapid decrease in $\tau_{c}$ around the pseudocritical temperature leads to a much stronger peak in the conductivity than the one observed at with a fixed value of $G$, as shown in Fig. 7(b). This prediction of the model can be tested by lattice QCD simulations.

\section{SUMMARY AND PERSPECTIVES}

Relativistic heavy collisions can produce strong magnetic fields. Strong magnetic fields have striking effects on properties of the hot quark matter created in such collisions. Measurability of many of the effects depend on the duration of the field produced in a collision. A key physical property determining the duration of the field is the electrical conductivity of the medium: the conductivity is responsible for the induction of electric currents which in turn can produce magnetic fields that can last while the systems exists. Simulations of the field dynamics involves solving relativistic magnetohydrodynamics equations which, for a realistic quantification require the temperature and magnetic field dependence of the electrical conductivity (and, of course, of other transport coefficients). We focused on the implications for the conductivity of changes induced by a magnetic field on the effective quark masses, with special interest on the importance of magnetic catalysis (MC) and inverse magnetic catalysis (IMC) - while MC occurs at low temperatures, IMC occurs at temperatures close to the pseudocritical temperature $\left(T_{\mathrm{pc}}\right)$ of the QCD phase transition. We employed a quasiparticle model for the medium, implemented by a Nambu-Jona-Lasinio model with a temperature- and magnetic-field-dependent coupling constant adjusted to lattice QCD data on MC and IMC. One of our main finding was that while the longitudinal conductivity $\left(\sigma^{z z}\right)$ increases with $B$, the transverse component $\left(\sigma^{x x}=\sigma^{y y}\right)$ decreases with $B$, in qualitative agreement with the very recent lattice QCD results of Ref. [19]. Moreover, our study revealed that IMC leaves a distinctive signal in all components of conductivity, a kink at $T_{\mathrm{pc}}$. In addition, IMC makes the peak more prominent in the quark and antiquark contributions to $\sigma^{x y}=-\sigma^{y x}$ at $e B \simeq 10 m_{\pi}^{2}$. Such a peak appears when the two timescales controlling the conductivity; i.e., the relaxation time $\tau_{c}$ and the inverse of the cyclotron frequency $\tau_{B}$ are comparable. This feature of IMC on these components is a prediction of the model and is testable with lattice QCD simulations. Additional findings in our study were (1) for a fixed value of $B$, all components of the conductivity increase with the temperature, (2) the anisotropy in the conductivity increases with $B$, (3) quantum effects leading to phase-space Landau-level quantization increase the longitudinal conductivity, and (4) truncation to the lowest Landau level gives a poor approximation for temperatures close to $T_{\mathrm{pc}}$.

Our study adds to the existing body of work on the hot quark matter electrical conductivity by incorporating nontrivial temperature and magnetic field effects on dynamical mass generation, such as MC and IMC. The results are useful for studies employing magnetohydrodynamics simulations of heavy-ion collisions and, on a wider perspective, they give insight on recent lattice QCD results on the electrical conductivity of magnetized hot quark matter.

\section{ACKNOWLEDGMENTS}

S. G. and J.D. acknowledge to IIT-Bhilai research facilities, funded by Ministry of Human Resource Development (MHRD), Government of India. Work partially supported by Conselho Nacional de Desenvolvimento Científico e Tecnológico - CNPq, Grants No. 304758/20175 (R. L. S. F), No. 305894/2009-9 (G. K.), and No. 464898/ 2014-5(G. K) (INCT Física Nuclear e Aplicações), and Fundação de Amparo à Pesquisa do Estado do Rio Grande do Sul - FAPERGS, Grant No. 19/2551-0000690-0 (R. L. S. F.), and Fundação de Amparo à Pesquisa do Estado de São Paulo - FAPESP, Grant No. 2013/01907-0 (G. K.) and Coordenação de Aperfeiçoamento de Pessoal de Nível Superior (CAPES) (A. B.) - Brasil (CAPES)- Finance Code 001. 
[1] J. Rafelski and B. Muller, Phys. Rev. Lett. 36, 517 (1976).

[2] D. E. Kharzeev, L. D. McLerran, and H. J. Warringa, Nucl. Phys. A803, 227 (2008).

[3] V. Skokov, A. Y. Illarionov, and V. Toneev, Int. J. Mod. Phys. A 24, 5925 (2009).

[4] K. Tuchin, Adv. High Energy Phys. 2013, 1 (2013).

[5] D. E. Kharzeev, J. Liao, S. A. Voloshin, and G. Wang, Prog. Part. Nucl. Phys. 88, 1 (2016).

[6] G. Wang and L. Wen, Adv. High Energy Phys. 2017, 9240170 (2017).

[7] J. Zhao and F. Wang, Prog. Part. Nucl. Phys. 107, 200 (2019).

[8] V. Voronyuk, V. D. Toneev, W. Cassing, E. L. Bratkovskaya, V. P. Konchakovski, and S. A. Voloshin, Phys. Rev. C 83, 054911 (2011).

[9] K. Tuchin, Phys. Rev. C 88, 024911 (2013).

[10] L. McLerran and V. Skokov, Nucl. Phys. A929, 184 (2014).

[11] U. Gursoy, D. Kharzeev, and K. Rajagopal, Phys. Rev. C 89, 054905 (2014).

[12] K. Tuchin, Phys. Rev. C 93, 014905 (2016).

[13] A. Amato, G. Aarts, C. Allton, P. Giudice, S. Hands, and J. I. Skullerud, Phys. Rev. Lett. 111, 172001 (2013).

[14] B. B. Brandt, A. Francis, H. B. Meyer, and H. Wittig, J. High Energy Phys. 03 (2013) 100.

[15] S. Gupta, Phys. Lett. B 597, 57 (2004).

[16] G. Aarts, C. Allton, J. Foley, S. Hands, and S. Kim, Phys. Rev. Lett. 99, 022002 (2007).

[17] H.-T. Ding, A. Francis, O. Kaczmarek, F. Karsch, E. Laermann, and W. Soeldner, Phys. Rev. D 83, 034504 (2011).

[18] P. V. Buividovich, M. N. Chernodub, D. E. Kharzeev, T. Kalaydzhyan, E. V. Luschevskaya, and M. I. Polikarpov, Phys. Rev. Lett. 105, 132001 (2010).

[19] N. Y. Astrakhantsev, V. V. Braguta, M. D’Elia, A. Y. Kotov, A. A. Nikolaev, and F. Sanfilippo, Phys. Rev. D 102, 054516 (2020).

[20] S. I. Nam, Phys. Rev. D 86, 033014 (2012).

[21] K. Hattori and D. Satow, Phys. Rev. D 94, 114032 (2016).

[22] K. Hattori, S. Li, D. Satow, and H.-U. Yee, Phys. Rev. D 95, 076008 (2017).

[23] A. Harutyunyan and A. Sedrakian, Phys. Rev. C 94, 025805 (2016).

[24] B. O. Kerbikov and M. A. Andreichikov, Phys. Rev. D 91, 074010 (2015).

[25] B. Feng, Phys. Rev. D 96, 036009 (2017).

[26] K. Fukushima and Y. Hidaka, Phys. Rev. Lett. 120, 162301 (2018).

[27] W. Li, S. Lin, and J. Mei, Phys. Rev. D 98, 114014 (2018).

[28] A. Das, H. Mishra, and R. K. Mohapatra, Phys. Rev. D 99, 094031 (2019).

[29] A. Das, H. Mishra, and R. K. Mohapatra, Phys. Rev. D 101, 034027 (2020).

[30] G. S. Bali, F. Bruckmann, G. Endrödi, Z. Fodor, S. D. Katz, S. Krieg, A. Schäfer, and K. K. Szabó, J. High Energy Phys. 02 (2012) 044.

[31] G. S. Bali, F. Bruckmann, G. Endrödi, Z. Fodor, S. D. Katz, and A. Schäfer, Phys. Rev. D 86, 071502(R) (2012).

[32] F. Bruckmann, G. Endrödi, and T. G. Kovacs, J. High Energy Phys. 04 (2013) 112.

[33] G. Endrödi, J. High Energy Phys. 04 (2013) 023.
[34] R. Gatto and M. Ruggieri, Lect. Notes Phys. 871, 87 (2013).

[35] V. A. Miransky and I. A. Shovkovy, Phys. Rep. 576, 1 (2015).

[36] J. O. Andersen, W. R. Naylor, and A. Tranberg, Rev. Mod. Phys. 88, 025001 (2016).

[37] Y. Nambu and G. Jona-Lasinio, Phys. Rev. 122, 345 (1961).

[38] Y. Nambu and G. Jona-Lasinio, Phys. Rev. 124, 246 (1961).

[39] U. Vogl and W. Weise, Prog. Part. Nucl. Phys. 27, 195 (1991).

[40] S. P. Klevansky, Rev. Mod. Phys. 64, 649 (1992).

[41] T. Hatsuda and T. Kunihiro, Phys. Rep. 247, 221 (1994).

[42] M. Buballa, Phys. Rep. 407, 205 (2005).

[43] R. L. S. Farias, K. P. Gomes, G. I. Krein, and M. B. Pinto, Phys. Rev. C 90, 025203 (2014).

[44] R. L. S. Farias, V. S. Timoteo, S. S. Avancini, M. B. Pinto, and G. Krein, Eur. Phys. J. A 53, 101 (2017).

[45] S. Li and H. U. Yee, Phys. Rev. D 97, 056024 (2018).

[46] S. Nam and C.-W. Kao, Phys. Rev. D 87, 114003 (2013).

[47] M. G. Alford, H. Nishimura, and A. Sedrakian, Phys. Rev. C 90, 055205 (2014).

[48] A. N. Tawfik, A. M. Diab, N. Ezzelarab, and A. G. Shalaby, Adv. High Energy Phys. 2016, 8 (2016).

[49] K. Tuchin, J. Phys. G 39, 025010 (2012).

[50] S. Ghosh, B. Chatterjee, P. Mohanty, A. Mukharjee, and H. Mishra, Phys. Rev. D 100, 034024 (2019).

[51] P. Mohanty, A. Dash, and V. Roy, Eur. Phys. J. A 55, 35 (2019).

[52] J. Dey, S. Satapathy, P. Murmu, and S. Ghosh, arXiv: 1907.11164.

[53] J. Dey, S. Satapathy, A. Mishra, S. Paul, and S. Ghosh, arXiv:1908.04335.

[54] A. Dash, S. Samanta, J. Dey, U. Gangopadhyaya, S. Ghosh, and V. Roy, Phys. Rev. D 102, 016016 (2020).

[55] K. Hattori, X. G. Huang, D. H. Rischke, and D. Satow, Phys. Rev. D 96, 094009 (2017).

[56] X.-G. Huang, M. Huang, D. H. Rischke, and A. Sedrakian, Phys. Rev. D 81, 045015 (2010).

[57] X. G. Huang, A. Sedrakian, and D. H. Rischke, Ann. Phys. (Amsterdam) 326, 3075 (2011).

[58] N. O. Agasian, Phys. At. Nucl. 76, 1382 (2013).

[59] N. O. Agasian, JETP Lett. 95, 171 (2012).

[60] D. Ebert and K. G. Klimenko, Nucl. Phys. A728, 203 (2003).

[61] D. P. Menezes, M. B. Pinto, S. S. Avancini, A. P. Martínez, and C. Providência, Phys. Rev. C 79, 035807 (2009).

[62] S. S. Avancini, R. L.S. Farias, M. Benghi Pinto, W. R. Tavares, and V. S. Timóteo, Phys. Lett. B 767, 247 (2017).

[63] S. S. Avancini, R. L. S. Farias, and W. R. Tavares, Phys. Rev. D 99, 056009 (2019).

[64] B. B. Brandt, G. Bali, G. Endrödi, and B. Glässle, Proc. Sci., LATTICE2015 (2016) 265 [arXiv:1510.03899].

[65] A. Ayala, M. Loewe, A. Z. Mizher, and R. Zamora, Phys. Rev. D 90, 036001 (2014).

[66] A. Ayala, M. Loewe, and R. Zamora, Phys. Rev. D 91, 016002 (2015).

[67] D. Son and B. Spivak, Phys. Rev. B 88, 104412 (2013).

[68] S. Ghosh, A. Lahiri, S. Majumder, R. Ray, and S. K. Ghosh, Phys. Rev. C 88, 068201 (2013).

[69] S. Ghosh, T. C. Peixoto, V. Roy, F. E. Serna, and G. Krein, Phys. Rev. C 93, 045205 (2016). 
[70] R. Lang and W. Weise, Eur. Phys. J. A 50, 63 (2014).

[71] R. Lang, N. Kaiser, and W. Weise, Eur. Phys. J. A 51, 127 (2015).

[72] P. Zhuang, J. Hufner, S. P. Klevansky, and L. Neise, Phys. Rev. D 51, 3728 (1995).

[73] P. Rehberg, S. P. Klevansky, and J. Hufner, Nucl. Phys. A608, 356 (1996).
[74] C. Sasaki and K. Redlich, Nucl. Phys. A832, 62 (2010).

[75] R. Marty, E. Bratkovskaya, W. Cassing, J. Aichelin, and H. Berrehrah, Phys. Rev. C 88, 045204 (2013).

[76] P. Deb, G. Kadam, and H. Mishra, Phys. Rev. D 94, 094002 (2016).

[77] S. Ghosh, F. E. Serna, A. Abhishek, G. Krein, and H. Mishra, Phys. Rev. D 99, 014004 (2019). 